\title{
On Problems Associated with Modeling Wing-Tail Configurations from Wind Tunnel Data
}

\author{
Patrick C. Murphy* \\ NASA Langley Research Center, Hampton, VA, 23681-2199 \\ Vladislav Klein ${ }^{\dagger}$ \\ The George Washington University, NASA Langley Research Center, Hampton, VA, 23681-2199
}

This paper considers factors that contribute to poor identification of unsteady aerodynamics from wind tunnel data for an airliner configuration. One approach to modeling a wing-tail configuration is considered and applied to both steady and largeamplitude forced pitch oscillation wind tunnel data taken over a wide range of angles of attack but a limited range of amplitude and frequencies. The identified models fit the measured data well but in some cases with inaccurate parameters. Only limited conclusions can be drawn from analysis of the current data set until further experiments can be performed to resolve the identification issues. The analysis of measured and simulated data provides some insights and guidance on how an effective experiment may be designed for wing-tail configurations with nonlinear unsteady aerodynamics.

\section{Nomenclature}

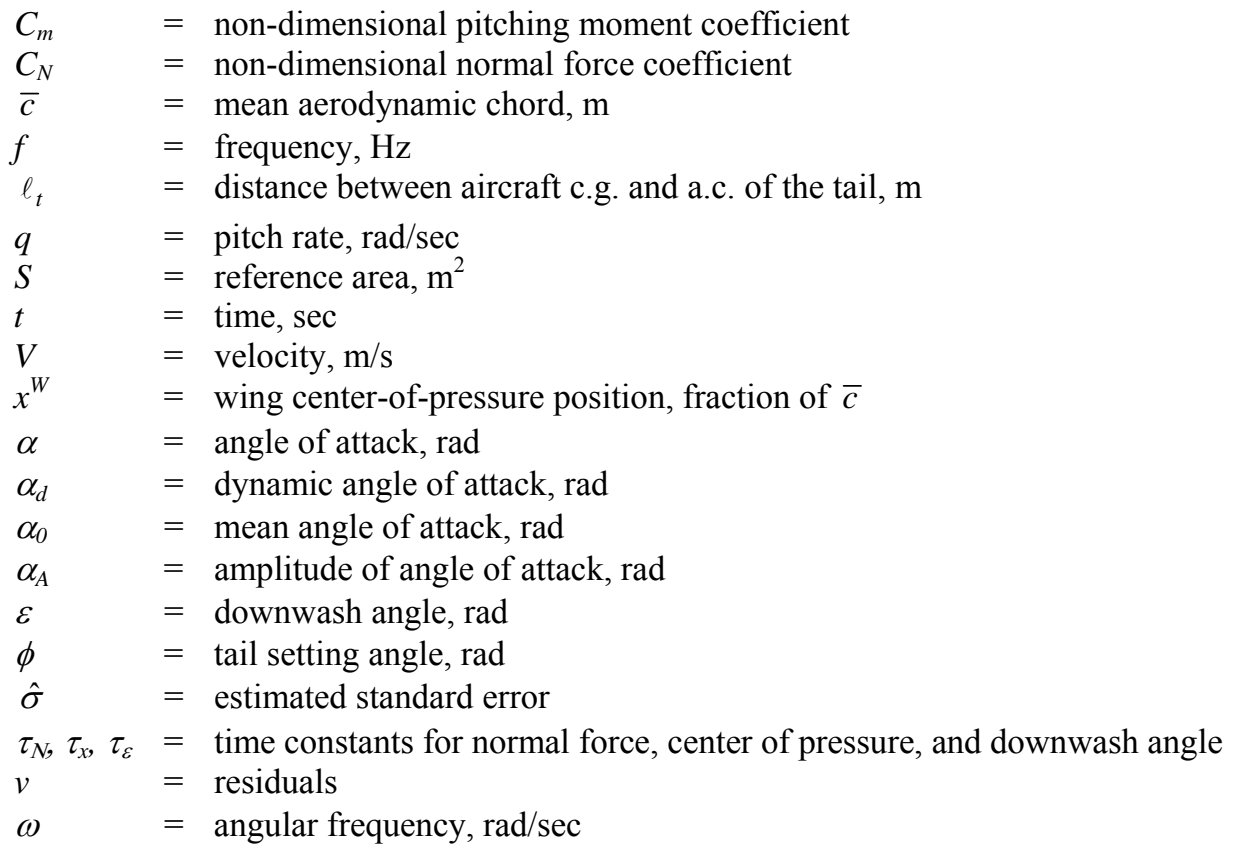

\footnotetext{
* Senior Research Engineer, Dynamic Systems \& Control Branch, Mail Stop 308, Associate Fellow.

${ }^{\dagger}$ Professor Emeritus, Dynamic Systems \& Control Branch, Mail Stop 308, Associate Fellow.
} 


$$
\begin{array}{ll}
\text { subscripts } & \\
\mathrm{A} & =\text { amplitude } \\
\mathrm{t} & =\text { tail }
\end{array}
$$

superscripts

$\mathrm{B}, \mathrm{T}, \mathrm{W}=$ body, tail, wing components

$\mathrm{BT}, \mathrm{BW}=$ body-tail, body-wing components
Aerodynamic Derivatives

$$
\begin{aligned}
C_{a_{\alpha}} & =\frac{\partial C_{a}}{\partial \alpha} \text { where } a=N \text { or } \mathrm{m} \\
C_{a_{q}} & =\frac{\bar{c}}{2 V} \frac{\partial C_{a}}{\partial a} \text { where } a=N \text { or } \mathrm{m}
\end{aligned}
$$

abbreviations

$\begin{array}{lll}\text { c.g. } & =\text { center of gravity } \\ \text { a.c. } & =\text { aerodynamic center }\end{array}$

\section{Introduction}

$\mathrm{O}$ ne of the first attempts to formulate aerodynamic model equations with unsteady terms based on wind tunnel data was reported in Ref. 1 and later in Ref. 2. The experimental data were in the form of forced oscillations in pitch with fixed amplitude and varied mean angle of attack and frequency. The test article was a simple delta wing. Later in the same decade, several reports and papers appeared with experiments including oscillations in roll and yaw, and with varied amplitudes ${ }^{3,4}$. Other planforms were also tested such as the F-16XL aircraft with cranked-delta wing $^{5}$ and the X-31 aircraft with delta wing and canard ${ }^{6}$.

Recently there has been increased interest in identification (model structure determination and parameter estimation) of a transport aircraft represented by a classical wing-tail configuration. The theoretical background for model formulation was given by Jones and Fehlner ${ }^{7,8}$ and Tobak $^{9}$. Later, Klein ${ }^{10}$ developed linear aerodynamic equations for planar motion of an aircraft with a horizontal tail. Unsteady effects in these equations were expressed by linear indicial functions for wing and tail aerodynamics and for the downwash angle.

A different model of the wing-tail combination was proposed by Khrabrov ${ }^{11}$ et. al. They assumed that the aircraft model can be represented by body, wing, and tail components and that the aerodynamic forces and moments are in general nonlinear and unsteady. The resulting model included algebraic and differential equations with parameters dependent upon the angle of attack. The model was used in the analysis of steady and unsteady wind tunnel data. The identified model was presented in several graphs comparing measured and estimated time histories. Unfortunately, no numerical values of estimated parameters and their accuracies were given.

Wind tunnel data and postulated model equations of Ref. 11 were used again in model identification and the results are summarized in Ref. 12. The identified models for different configurations mostly fit the measured data quite well. Some of the estimated parameters, however, were estimated with low accuracy resulting in non-physical values. There were no a priori values of the parameters and no additional measured data which could be used for model validation.

The purpose of this paper is to re-examine the results of previous data analysis addressing mainly measured data inconsistency, low accuracy and non-physical values of parameter estimates, and model adequacy. This will provide the groundwork and direction to investigate appropriate experiment designs that can allow identification of wing-tail configurations that exhibit nonlinear unsteady dynamics. In addition, a proposal for future experiment design for obtaining wind tunnel data with high accuracy and high information content will be outlined. The experiment should provide steady data for all configurations, small amplitude oscillatory data for estimating damping parameters, large amplitude oscillatory data for model identification, and specific data for checking model adequacy and prediction capability.

\section{Measured Data}

The measured data were obtained from static and dynamic testing of an airliner in the Central AeroHydrodynamic Institute (TsAGI) low subsonic wind tunnel ${ }^{11}$. The steady data included the normal force and the pitching moment of four different configurations, i.e., body alone (B), body-tail (BT), body-wing (BW) and the complete model (BWT). These measurements cover the angles of attack from $-10^{\circ}$ up to $30^{\circ}$.

The dynamic data were obtained from a forced oscillatory motion of the same model in the same tunnel using the same test rig. The experiment was executed at different initial values of angle of attack, $\alpha_{0}$, frequencies, and amplitudes, $\alpha_{\mathrm{A}}$. For aircraft model identification, large amplitude $\left(\alpha_{\mathrm{A}}>5^{\circ}\right)$ time histories of $C_{N}$ and $C_{m}$ for three configurations BT, BW, and BWT, at frequencies approximately equal to $0.4 \mathrm{~Hz}, 0.8 \mathrm{~Hz}$, and $1.3 \mathrm{~Hz}$, were used. For large oscillation amplitudes of $15^{\circ}, \alpha_{0}$ was equal to $5^{\circ}$ and $15^{\circ}$, and for oscillation amplitudes of $10^{\circ}$, $\alpha_{0}$ was equal to $5^{\circ}, 10^{\circ}$, and $20^{\circ}$. Only one cycle of oscillation was given in each time history. 


\section{Models for Steady Aerodynamics}

The aerodynamic forces and moments of the complete aircraft are assumed to be the sum of separate contributions from the body, tail, and wing ${ }^{11}$. Then the corresponding models for the steady case have the form

$$
\begin{gathered}
C_{N}(\alpha)=C_{N}^{B}(\alpha)+C_{N}^{W}(\alpha)+\bar{S}_{t} C_{N}^{T}\left(\alpha_{t}\right) \\
C_{m}(\alpha)=C_{m}^{B}(\alpha)+C_{m_{0}}^{W}+C_{N}^{W}(\alpha) x^{W}(\alpha)-\bar{\ell}_{t} \bar{S}_{t} C_{N}^{T}\left(\alpha_{t}\right)
\end{gathered}
$$

where

$$
\alpha_{t}=\alpha+\phi-\varepsilon(\alpha)
$$

$\bar{S}_{t}=S_{t} / S, \bar{\ell}_{t}=\ell_{t} / \bar{C}$, and $x^{W}$ is the distance between the wing center of pressure and aircraft c.g. as a fraction of mean aerodynamic chord. The static values of $C_{N}$ and $C_{m}$ for a complete model and its components are shown in Fig. 1 and 2.

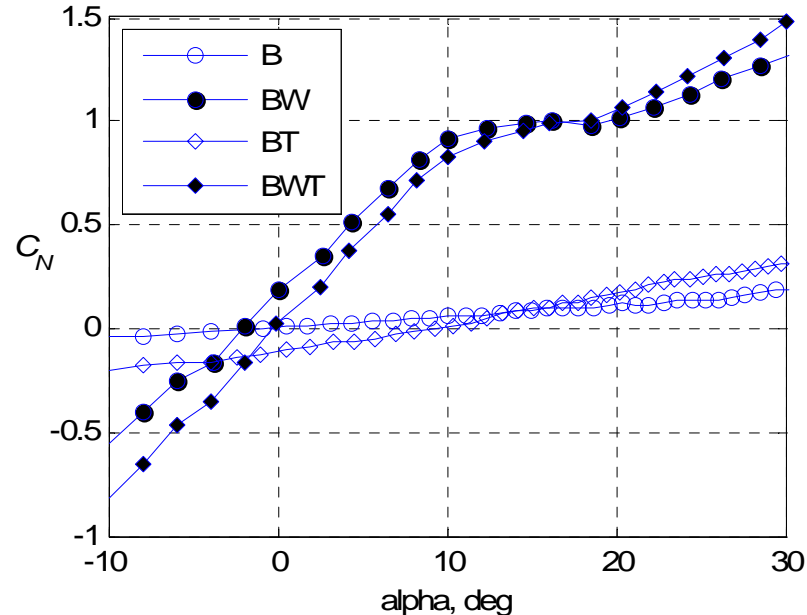

Figure 1. Normal-force coefficient for four configurations (Ref. 11).

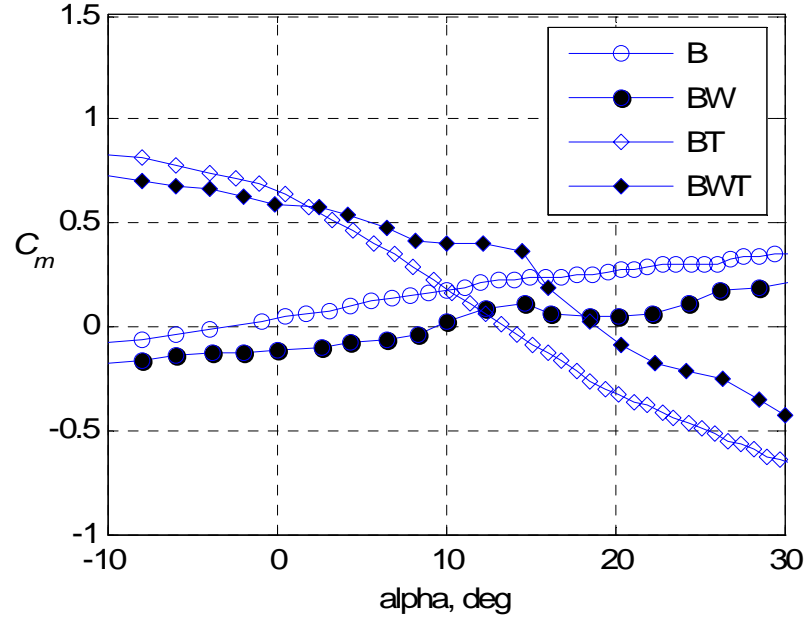

Figure 2. Pitching-moment coefficient for four configurations (Ref. 11).

In these plots B, BW, BT, and BWT indicate the values for the body, body-wing, body-tail, and the complete model. The values of $C_{N}^{W}$ and $C_{N}^{T}$ in Eq. (1) and Eq. (2) were obtained from the relations

$$
\begin{gathered}
C_{N}^{W}=C_{N}^{B W}-C_{N}^{B} \\
C_{N}^{T}=C_{N}^{B T}-C_{N}^{B}
\end{gathered}
$$

In Eqs. (1-3), $C_{m_{0}}^{W}$ is equal to the wing pitching moment when $C_{N}^{W}$ is equal to zero, $\phi$ is the tail plane setting and $\varepsilon$ is the downwash angle at the tail. From the geometry of the model $\bar{S}_{t}=0.26, \bar{\ell}_{t}=4.62$ and $\phi=-10^{\circ}$ and from $C_{m}^{W}$ the value of $C_{m_{0}}^{W}=-0.13$.

The variable $x^{W}(\alpha)$ can be computed by removing terms associated with the tail in Eq. (2) and using the corresponding BW measurements. The downwash angle follows from solving Eqs. (1-3) for $\alpha_{t}$ at selected $\alpha$. The two variables, $x^{W}(\alpha)$ and $\varepsilon(\alpha)$, are plotted in Figs. 3 and 4 and compared with results from Ref. 11. The differences between the two pairs are small. 


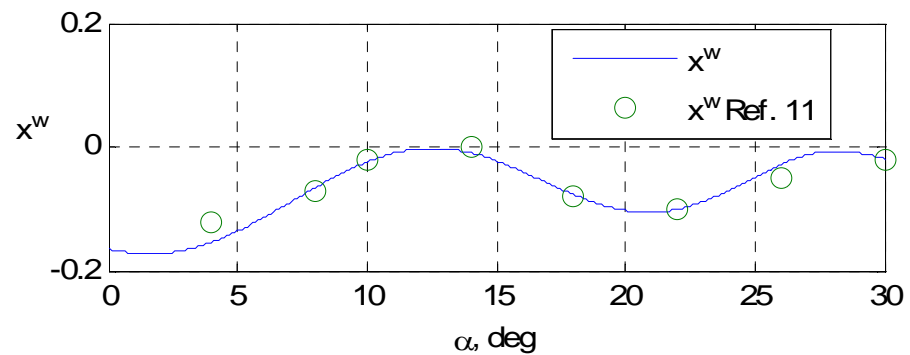

Figure 3. Distance $x^{w}$ estimated from steady wind tunnel data.

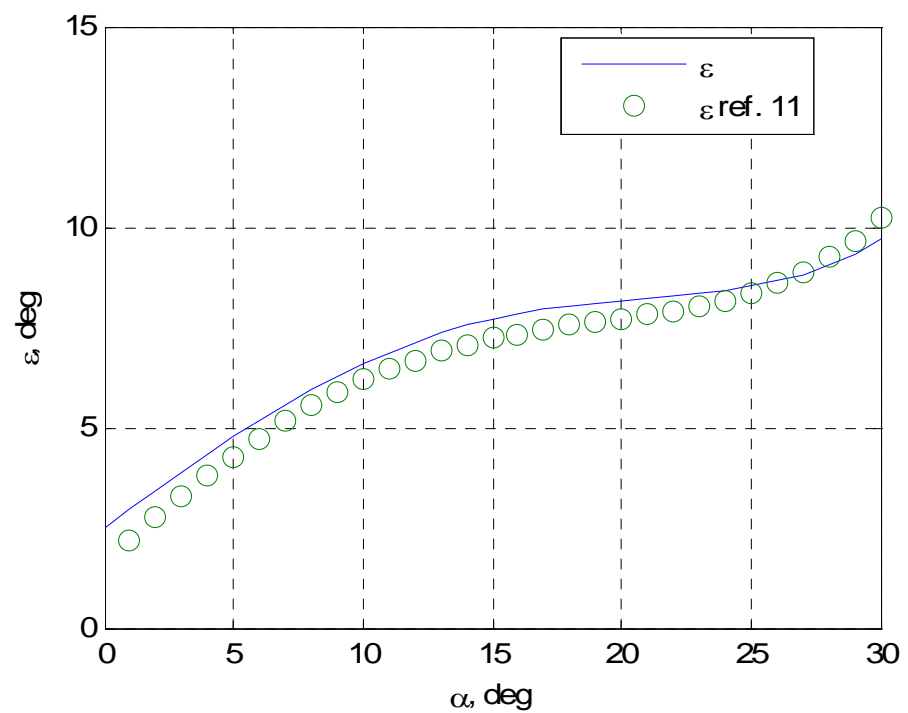

Figure 4. Downwash angle at the tail estimated from steady wind tunnel data.

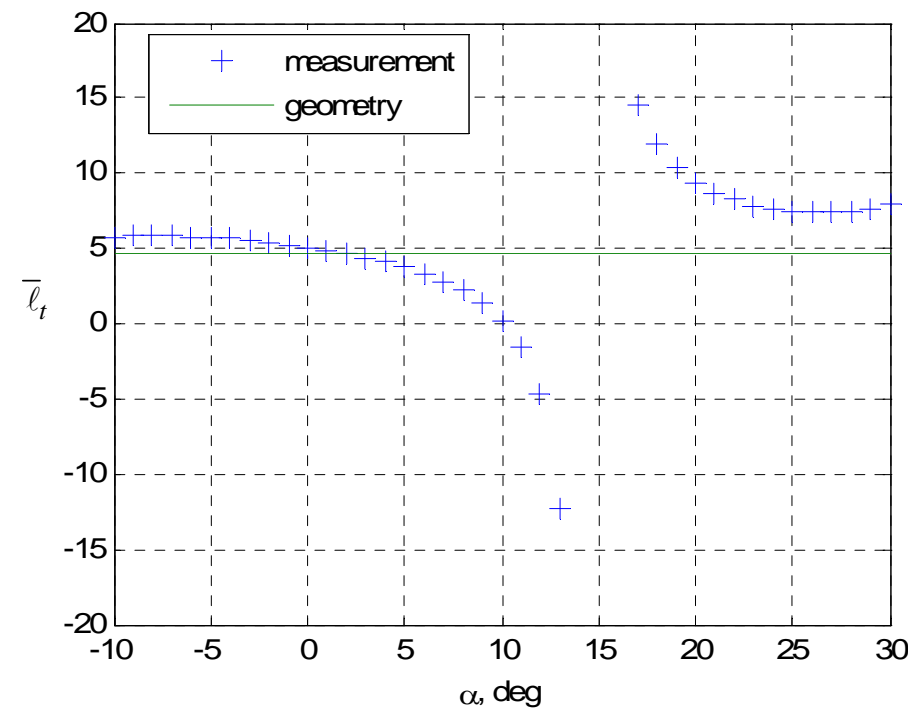

Figure 5. Tail length determined from aircraft geometry and from tail coefficient measurements.

In pitching-moment Eq. (2) the last term $\left\{-\bar{\ell}_{t} \bar{S}_{t} C_{N}^{T}\left(\alpha_{t}\right)\right\}$ represents the tail contribution, $C_{m}^{T}\left(\alpha_{t}\right)$. In order to determine the best representation for this term, two evaluations of the consistency between the measured normal 
force and pitching moment were made. First, both $C_{m}^{T}\left(\alpha_{t}\right)$ and $-\bar{\ell}_{t} \bar{S}_{t} C_{N}^{T}\left(\alpha_{t}\right)$ terms were used to compute the tail length as

$$
\bar{\ell}_{t}=-\frac{C_{m}^{T}}{\bar{S}_{t} C_{N}^{T}}
$$

and plotted against $\alpha$ in Fig. 5. Changes in $\bar{\ell}_{t}$ with respect to $\alpha$ should be relatively small. Large values of computed $\bar{\ell}_{t}$ around $\alpha=15^{\circ}$ are mainly caused by $C_{N}^{T}$ approaching zero, see Fig 1 . For $\alpha>20^{\circ}$, the differences between $\bar{\ell}_{t}$ computed and its nominal value are larger than expected, indicating one contributor to data inconsistency may result from inaccuracies of measured $C_{m}^{T}$ and $C_{N}^{T}$.

The second check of data consistency is based on a comparison of normal force and pitching moment of the complete aircraft with that computed from separate measurements of the body, body-tail, and body-wing. For complete consistency, the following relation applies.

$$
C_{a}^{B W T}=C_{a}^{B}+C_{a}^{T}+C_{a}^{W}
$$

where subscript $a$ is either $N$ or $m$. Using Eqs. (4) and (5) the relation in Eq. (7) can be written as

$$
C_{a}^{B W T}=C_{a}^{B T}+C_{a}^{B W}-C_{a}^{B}
$$

A check of the pitching moment equation can also be made by replacing $C_{m}^{T}$ in Eq. (7) by $-\bar{\ell}_{t} \bar{S}_{t} C_{N}^{T}$, giving

$$
C_{m}^{B W T}=C_{m}^{B W}-\bar{\ell}_{t} \bar{S}_{t} C_{N}^{T}
$$

Figure 6 shows the comparison of measured $C_{a}^{B W T}$ with that computed using Eq. (8) for both normal force and pitching moment and Eq. (9) for pitching moment. Normal force comparisons demonstrate very good agreement between measured and computed data. Based on comparisons shown in Figs. 5 and 6, one can expect better accuracy of measured $C_{N}^{T}$ than $C_{m}^{T}$. Consequently, Eq. (2) is used in this study as stated.

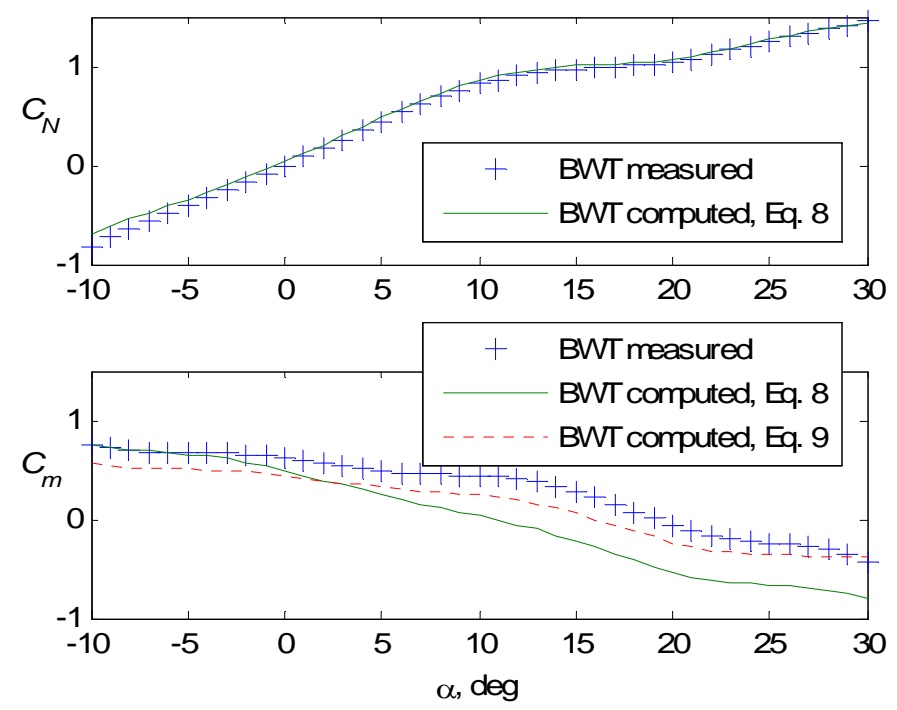

Figure 6. Measured and computed coefficients for complete aircraft .

American Institute of Aeronautics and Astronautics 


\section{Analysis of Large Amplitude Oscillatory Data}

The aerodynamic model equations for modeling and data analysis are given in Refs. 11 and 12. They are repeated here for convenience.

$$
\begin{gathered}
C_{N}(t ; \alpha)=C_{N}^{B}(\infty ; \alpha)+C_{N}^{W}(t ; \alpha)+\bar{S}_{t} C_{N}^{T}\left(t ; \alpha_{t}\right)+\frac{\bar{c}}{2 V} C_{N q}(\infty ; \alpha) \dot{\alpha}(t) \\
C_{m}(t ; \alpha)=C_{m}^{B}(\infty ; \alpha)+C_{m_{0}}^{W}+C_{N}^{W}(t ; \alpha) x^{W}(t ; \alpha)-\bar{\ell}_{t} \bar{S}_{t} C_{N}^{T}\left(t ; \alpha_{t}\right)+\frac{\bar{c}}{2 V} C_{m q}(\infty ; \alpha) \dot{\alpha}(t)
\end{gathered}
$$

where

$$
\begin{gathered}
\alpha_{t}=\alpha+\phi+\alpha_{d}-\varepsilon(t ; \alpha) \\
\alpha_{d}=\frac{\ell_{t} \dot{\alpha}}{V} \\
\tau_{N}(\alpha) \dot{C}_{N S}^{W}+C_{N S}^{W}=C_{N S}^{W}(\infty ; \alpha) \\
\tau_{x}(\alpha) \dot{x}^{W}+x^{W}=x^{W}(\infty ; \alpha) \\
\tau_{\varepsilon}(\alpha) \dot{\varepsilon}+\varepsilon=\varepsilon(\infty ; \alpha)
\end{gathered}
$$

The normal force coefficient due to the wing is partitioned as

$$
C_{N}^{W}=C_{N A}^{W}+C_{N S}^{W}
$$

where $C_{N A}^{W}$ is the linear part (attached flow) and $C_{N S}^{W}$ is the nonlinear part (separated flow). Symbol $\infty$ indicates a variable evaluated in steady flow. Unknown parameters in the model are the time constants, $\tau_{N}, \tau_{x}, \tau_{\varepsilon}$, and two damping terms, $C_{N_{q}}(\infty ; \alpha)$ and $C_{m_{q}}(\infty ; \alpha)$. In general, each of these parameters can be a function of $\alpha$. Whenever necessary these equations were modified to allow estimation with better accuracy.

\section{A. BT Configuration}

The model equations for the BT configuration follow from Eqs (10-13) as

$$
\begin{gathered}
C_{N}(t)=C_{N}^{B}(\alpha)+\bar{S}_{t} C_{N}^{T}\left(\alpha_{t}\right)+C_{N q}^{T}(\alpha) \frac{\dot{\alpha} \bar{c}}{2 V} \\
C_{m}(t)=C_{m}^{B}(\alpha)-\bar{\ell}_{t} \bar{S}_{t} C_{N}^{T}\left(\alpha_{t}\right)+C_{m q}^{T}(\alpha) \frac{\dot{\alpha} \bar{c}}{2 V} \\
\alpha_{t}=\alpha+\phi+\alpha_{d} .
\end{gathered}
$$

where $\alpha_{d}$ reflects a tail angle-of-attack increment due to rotation given by Eq. (13).

In these equations the only unknowns are the damping parameters, $C_{N q}^{T}$ and $C_{m q}^{T}$. Using the data for $\alpha_{0}=5^{\circ}, \alpha_{0}$ $=5^{\circ}$, and $f=0.4 \mathrm{~Hz}$, and assuming that the parameters are not $\alpha$ dependent, the following least squares estimates and their standard errors were obtained as

$$
C_{N q}^{T}=6.5 \pm 1.7 \text { and } C_{m q}^{T}=-21 \pm 30
$$


with the coefficient of determination, $\mathrm{R}^{2}$, close to zero. These results suggest inaccurate measured data and/or modeling errors in Eqs. (18 or 19).

To find the reason for such high parameter inaccuracy and low $\mathrm{R}^{2}$ values, the residuals $v_{N}$ and $v_{m}$ were computed from Eqs. (18-19), respectively and examined. Plots of the residuals against time and $\alpha$ are presented in Figs. 7 and 8.
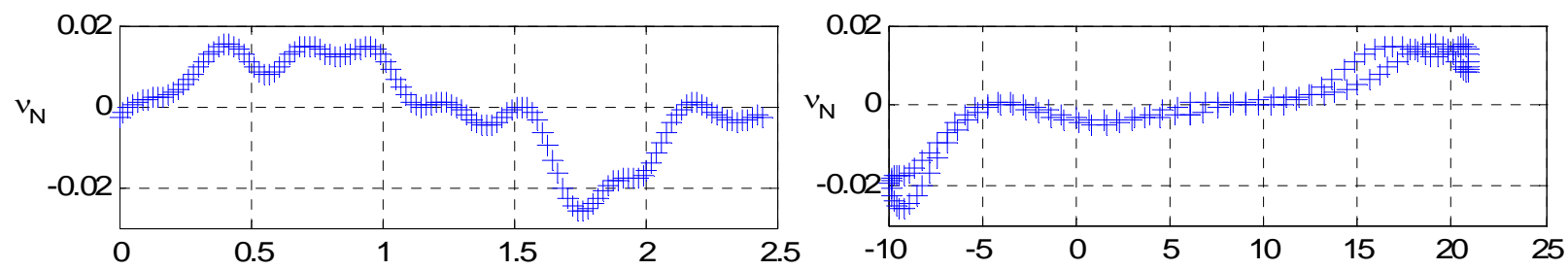

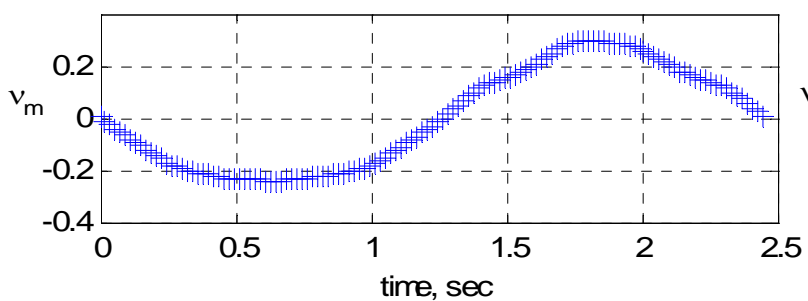

Figure 7. Residuals for model Eqs. (18-19) as a function of time.

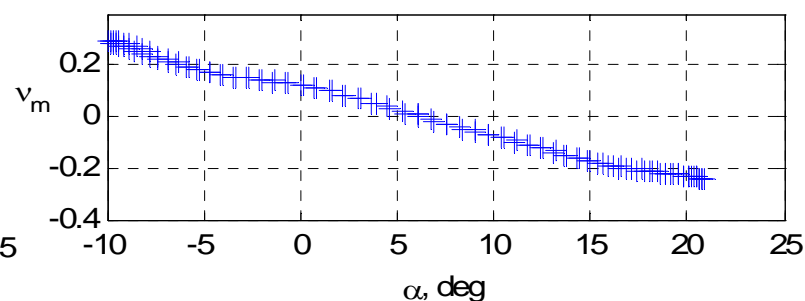

Figure 8. Residuals for model Eqs. (18-19) as a function of angle of attack.

From these plots the dependence of residuals on $\alpha$ is evident. For that reason additional terms were added to the model equations yielding

$$
\begin{gathered}
C_{N}(t)=C_{N}^{B}(\alpha)+\bar{S}_{t} C_{N}^{T}\left(\alpha_{t}\right)+\Delta_{N_{\alpha}} \alpha+C_{N q}^{T}(\alpha) \frac{\dot{\alpha} \bar{c}}{2 V} \\
C_{m}(t)=C_{m}^{B}(\alpha)-\bar{\ell}_{t} \bar{S}_{t} C_{N}^{T}\left(\alpha_{t}\right)+\Delta_{m_{\alpha}} \alpha+C_{m q}^{T}(\alpha) \frac{\dot{\alpha} \bar{c}}{2 V}
\end{gathered}
$$

The new parameter estimates in Eqs. (21-22) were obtained as

$$
\begin{array}{|l|l|}
\hline C_{N q}^{T}=6.5 \pm 0.73 & C_{m q}^{T}=-21 \pm 2.0 \\
\hline \Delta_{N_{\alpha}}=0.053 \pm 0.0022 & \Delta_{m_{\alpha}}=-1.006 \pm 0.0059 \\
\hline R^{2}=0.84 & R^{2}=0.99 \\
\hline
\end{array}
$$

A comparison of results indicates the following:

a) The mean values of damping parameters remain the same because in harmonic motion $\alpha$ and $q$ are orthogonal.

b) The use of a new model resulted in a dramatic improvement of parameter accuracy and model adequacy.

c) Estimated bias terms, $\Delta_{N_{\alpha}}$ and $\Delta_{m_{\alpha}}$, are close to a linear approximation of the slope for $v_{N}$ and $v_{m}$, see Fig. 8.

For the remaining discussion, the estimates will be based on Eqs. (21-22). 

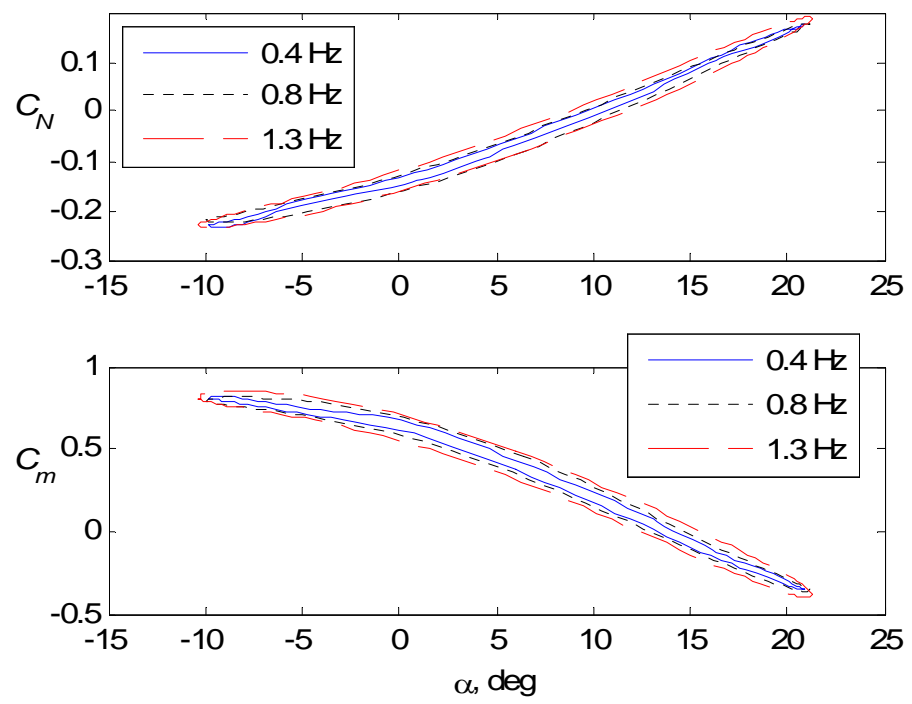

Figure 9. Measured normal-force and pitching moment coefficients for three frequencies at $\alpha_{0}=5^{\circ}$ and $\alpha_{\mathrm{A}}=15^{\circ}$.

For an assessment of frequency dependence, the measured $C_{N}$ and $C_{m}$ for three frequencies were plotted in Fig. 9. In this figure a minor effect of frequency can be seen. Its significance was evaluated by a comparison of two sets of estimates containing:

a) Average values of damping parameters estimated from three sets of data each with a single frequency,

$$
C_{N q}^{T}=5.9 \pm 0.42, C_{m q}^{T}=-18.7 \pm 1.2
$$

b) Estimated parameters from a combined set of data with all three frequencies included,

$$
C_{N q}^{T}=5.5 \pm 0.18, C_{m q}^{T}=-17.6 \pm 0.53
$$

Because of statistically insignificant differences in the two sets considered it was decided to use the combined data including all three frequencies. The final estimates and standard errors for five different test conditions are summarized in Table I.

\begin{tabular}{|c|c|c|c|c|c|c|c|}
\hline \multicolumn{2}{|c|}{ Table I. Estimated damping parameters from wind tunnel data for BT configuration } \\
\hline \multirow{2}{*}{$\begin{array}{c}\alpha_{0} \\
(\mathrm{deg})\end{array}$} & $\begin{array}{c}\alpha_{\mathrm{A}} \\
(\mathrm{deg})\end{array}$ & $C_{N q}^{T}$ & $\hat{\sigma}$ & $R^{2}$ & $C_{m q}^{T}$ & $\hat{\sigma}$ & $R^{2}$ \\
\hline 5 & 15 & 5.5 & 0.18 & 0.86 & -17.6 & 0.53 & 0.99 \\
\hline 15 & 15 & 5.4 & 0.22 & 0.78 & -15.5 & 1.30 & 0.96 \\
\hline 5 & 10 & 5.4 & 0.23 & 0.70 & -15.4 & 0.60 & 0.99 \\
\hline 10 & 10 & 4.7 & 0.26 & 0.83 & -13.7 & 0.85 & 0.99 \\
\hline 20 & 10 & 4.7 & 0.37 & 0.30 & -16.6 & 0.85 & 0.86 \\
\hline
\end{tabular}

Damping parameters in Table I are compared to the out-of-phase components of small amplitude $\left(\alpha_{A}=3^{\circ}\right)$ oscillatory data presented in Figs. 10 and 11. These components are expressed as

$$
\bar{C}_{a_{q}}=C_{a_{q}}+C_{a \dot{\alpha}} \quad a=N \text { or } m
$$

where $C_{N \dot{\alpha}}$ and $C_{m \dot{\alpha}}$ are constants for data without frequency effect or they equate to their unsteady counterparts when frequency dependence is present ${ }^{5}$. In the given experiment, frequency dependence is noticeable for $\bar{C}_{a_{q}}$ where $\alpha_{0}>10^{\circ}$.

Assuming that the tail is the main contributor to damping, the out-of-phase components can be formulated ${ }^{13}$ as 


$$
\bar{C}_{a_{q}}=C_{a_{q}}\left(1+\frac{\partial \varepsilon}{\partial \alpha}\right) \quad a=N \text { or } m
$$

From Fig. 4, $\partial \varepsilon / \partial \alpha \approx 0.5$, is representative of the slope of $\varepsilon(\alpha)$ near $\alpha_{0}=0$. Furthermore, from Fig. 10 and 11 , $\bar{C}_{N_{q}}=12.9$ and $\bar{C}_{m_{q}}=-37.9$, for $-4^{\circ}<\alpha<4^{\circ}$ and all frequencies. Therefore from Eq. (24) the damping parameters can be obtained as $C_{N_{q}}=8.6$ and $C_{m_{q}}=-25.3$. Both values are higher than those in Table I. The reason for these differences could be due to a comparison of results from small and large amplitude oscillatory data and partly due to Table I values not containing any contribution to damping from the wing.

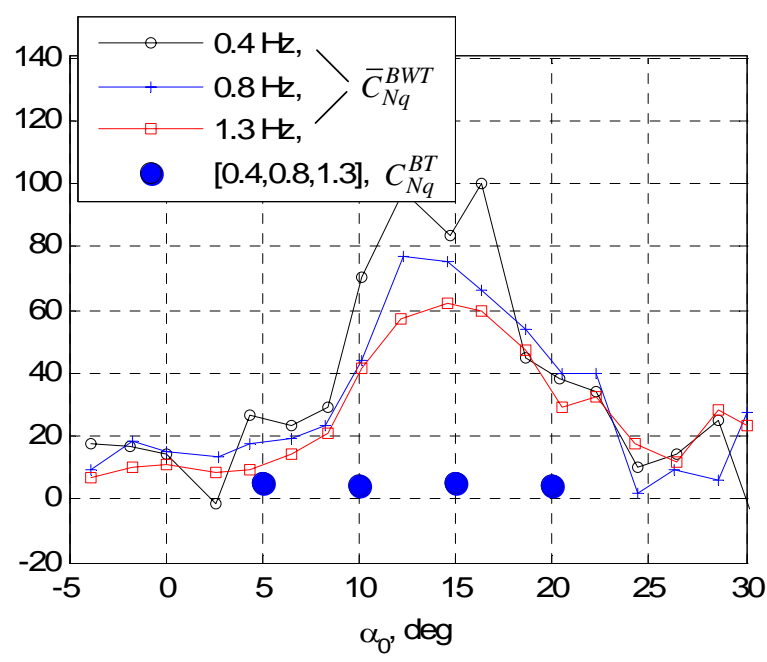

Figure 10. Estimated normal-force damping coefficients due to pitch rate from oscillatory data for BT and BWT configurations.

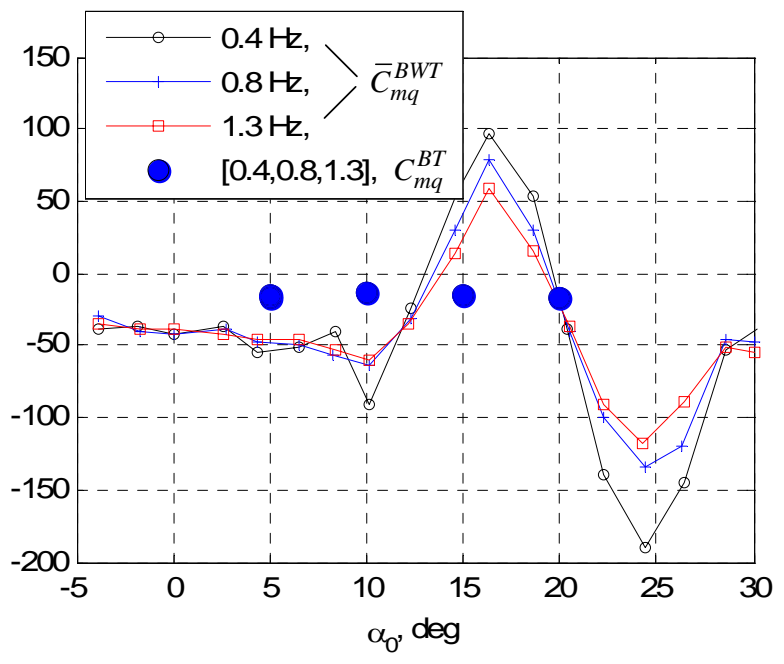

Figure 11. Estimated pitch damping coefficients from oscillatory data for BT and BWT configurations.

Rigorous estimation of the out-of-phase components at different $\alpha_{0}$ can be obtained from small amplitude oscillatory data at more than five carefully selected frequencies ${ }^{5}$. These results can provide first indication of the damping magnitude and its variation with angle of attack.

\section{B. BW Configuration}

The BW model equations follow from the general model in Eqs. (10-15) as

$$
\begin{gathered}
C_{N}(t)=C_{N}^{B}(\infty ; \alpha)+C_{N}^{W}(t ; \alpha)+C_{N q}^{B W}(\infty ; \alpha) \frac{\dot{\alpha} \bar{c}}{2 V} \\
C_{m}(t)=C_{m}^{B}(\infty ; \alpha)+C_{m_{0}}^{W}+C_{N}^{W}(t ; \alpha) x^{W}(\alpha)+C_{m q}^{B W}(\infty ; \alpha) \frac{\dot{\alpha} \bar{c}}{2 V}
\end{gathered}
$$

As in the complete model, the normal force coefficient was partitioned as

$$
C_{N}^{W}=C_{N A}^{W}+C_{N S}^{W}
$$

then a time delay due to flow separation can be estimated from

$$
\tau_{N}(\alpha) \dot{C}_{N S}^{W}+C_{N S}^{W}=C_{N S}^{W}(\infty ; \alpha)
$$


Similarly, for identification of the pitching-moment model, the coordinate of the wing center of pressure can be estimated from

$$
\tau_{x}(\alpha) \dot{x}^{W}+x^{W}=x^{W}(\infty ; \alpha)
$$

Now the estimation problem for the $C_{N}$ model parameters can be formulated as follows: given time histories of $C_{N}(t), \alpha(t)$, and $\dot{\alpha}(t)$, and analytical functions $C_{N S}^{W}(\infty ; \alpha), C_{N A}^{W}(\infty ; \alpha)$, and $C_{N}^{B}(\infty ; \alpha)$, estimate unknown constants $\tau_{N}$ and $C_{N q}^{B W}$. Using simplified notation, $\eta=C_{N S}^{W}, \quad \theta_{1}=\tau_{N}, \quad \theta_{2}=\frac{\bar{c}}{2 V} C_{N q}^{B W}, \quad u=C_{N S}^{W}(\infty ; \alpha)$, $y=C_{N}(t)-C_{N}^{B}(\infty ; \alpha)-C_{N A}^{W}(\infty ; \alpha)$, then

$$
\begin{aligned}
& \theta_{1} \dot{\eta}+\eta=u \\
& y=\eta+\theta_{2} \dot{\alpha}
\end{aligned}
$$

A similar formulation can be made for $C_{m}$ where the unknown parameters to be estimated are $\tau_{x}$ and $C_{m q}^{B W}$. The unknown parameters in the state-space models were estimated by an output error method explained in Ref. 4.

Parameter estimates and their standard errors are given in Table II. Both time constant and damping parameters were considered independent of $\alpha$ for each data set analyzed. The average value of $\tau_{N} \approx 13$ for all five cases agrees with limited previous experience. The estimates of the second time constant exhibit an increase with increased angle of attack. These values could not be verified because no a priori information on them existed.

Table II. Estimated damping parameters and non-dimensional time constants from large-amplitude oscillatory data, BW configuration.

\begin{tabular}{|c|c|rl|rl|rl|r|r|}
\hline$\alpha_{0}, \operatorname{deg}$ & \multirow{2}{*}{$\alpha_{A}, \operatorname{deg}$} & \multicolumn{2}{|c|}{$\tau_{N}$} & \multicolumn{2}{|c|}{$C_{N q}^{B W}$} & \multicolumn{2}{|c|}{$\tau_{x}$} & \multicolumn{2}{|c|}{$C_{m q}^{B W}$} \\
\hline & & \multicolumn{1}{|c|}{$\hat{\theta}$} & $(\hat{\sigma})$ & \multicolumn{1}{|c|}{$\hat{\theta}$} & $(\hat{\sigma})$ & \multicolumn{1}{|c|}{$\hat{\theta}$} & $(\hat{\sigma})$ & $\hat{\theta}$ & $(\hat{\sigma})$ \\
\hline 5 & 15 & $12.5(1.2)$ & 4.8 & $(2.2)$ & 50.0 & $(3.2)$ & $5.1(.63)$ \\
\hline 15 & 15 & $9.6(1.4)$ & 8.8 & $(3.7)$ & 40.6 & $(2.6)$ & $7.4(.78)$ \\
\hline 5 & 10 & $14.4(1.9)$ & $5.8(2.9)$ & 25.6 & $(1.7)$ & $3.8(.71)$ \\
\hline 10 & 10 & $12.7(1.6)$ & $10.5(4.0)$ & 34.0 & $(1.6)$ & $10.5(.69)$ \\
\hline 20 & 10 & $16.6(1.4)$ & 13.7 & $(4.4)$ & 56.2 & $(.67)$ & $0.2(.67)$ \\
\hline
\end{tabular}

Damping parameters in Table II were compared in Figs. 12 and 13 with the estimates from small amplitude oscillatory data. For the low $\alpha$ region, the out-of-phase components are approximately $\bar{C}_{N q}^{B W} \cong 5$ and $\bar{C}_{m q}^{B W} \cong-3$. This implies that $C_{N q}^{B W}$ estimates in Table II are very high and that $C_{m q}^{B W}$ estimates are incorrect due to their nonphysical values. The reason for these discrepancies was found in the low sensitivity of responses $C_{N}(t)$ and $C_{m}(t)$ to both damping parameters. This insensitivity was demonstrated computing these responses and their residuals using estimated $\tau_{N}$ and $\tau_{x}$ values along with appropriately specified values of $C_{N q}^{B W}$ and $C_{m q}^{B W}$. The results, in each case, indicated almost no change in the responses and residuals. 


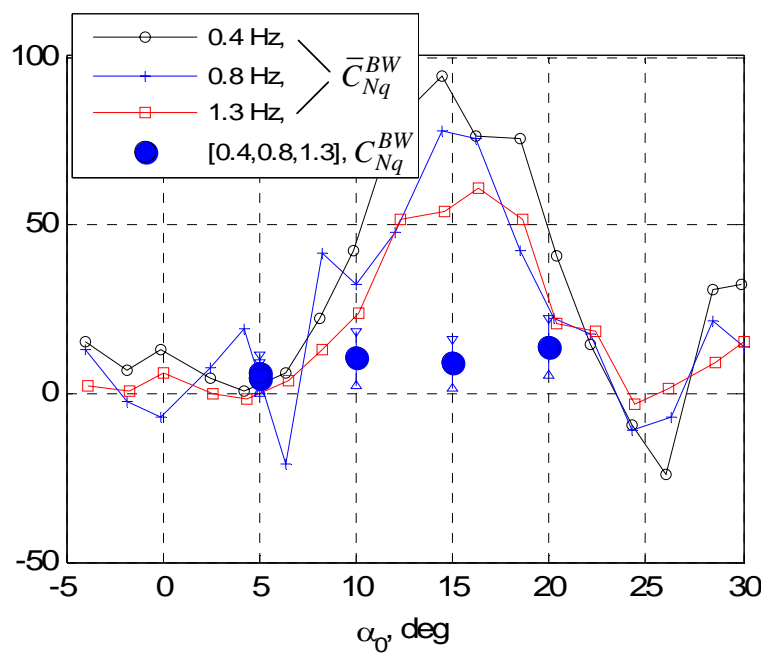

Figure 12. Estimated normal-force damping coefficients due to pitch rate from oscillatory data for BW configuration.

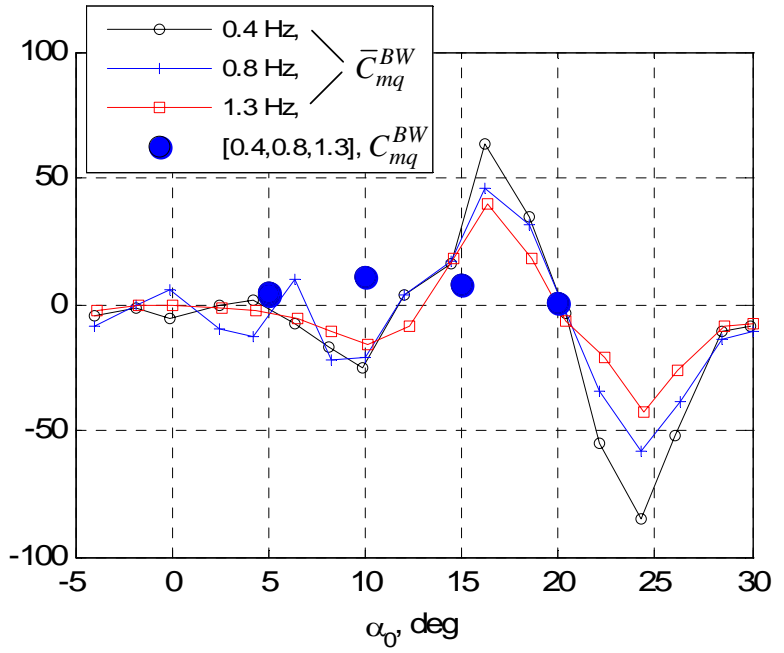

Figure 13. Estimated pitch damping coefficients from oscillatory data for BW configuration.

Measured and computed time histories of $C_{N}$ and $C_{m}$ using parameter values from Table II are plotted in Figs. 14 and 15. Although forced-oscillation experiments were performed separately for each frequency, time history plots show all 3 frequency cases stacked into one plot for analysis. In general, there is good agreement between these plots. Some effect of uncorrected model error in $C_{m}$ is visible in part of the data with $f=0.4 \mathrm{~Hz}$. Figs. 16 and 17 show both coefficients plotted against $\alpha$ with $f=0.8 \mathrm{~Hz}$. Included in both figures are also steady values of both coefficients. Part of the $C_{N}(\alpha)$ curve for $10^{\circ}<\alpha<20^{\circ}$ with pronounced unsteady effect is captured quite well by identified model despite inaccuracies in both damping parameters. Unexplained differences, however, are apparent in the linear part of $C_{N}(\alpha)$. The agreement between measured and computed $C_{m}(\alpha)$ is poor. Finding an explanation for this requires additional study.

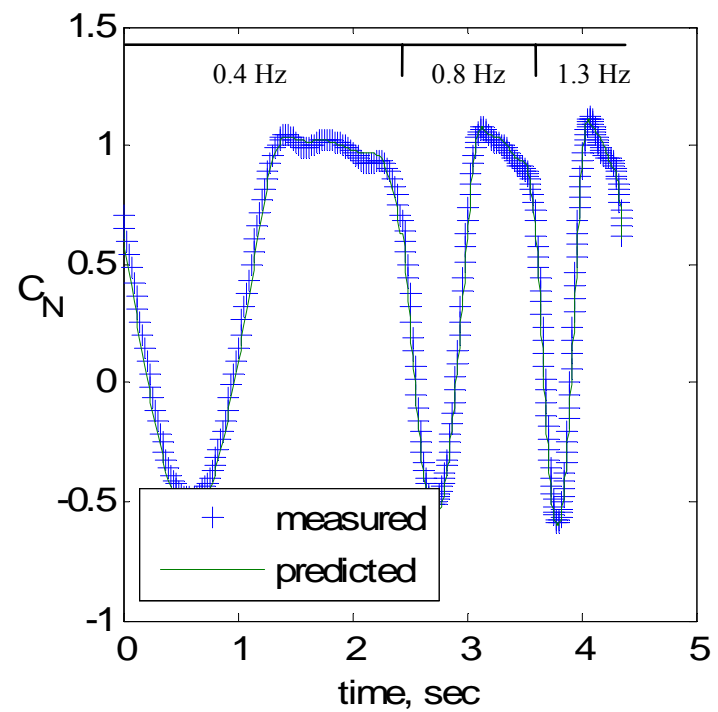

Figure 14. Measured and computed time histories of normal force for BW configuration, $\alpha_{0}=5^{\circ}$, and $\alpha_{A}=15^{\circ}$.

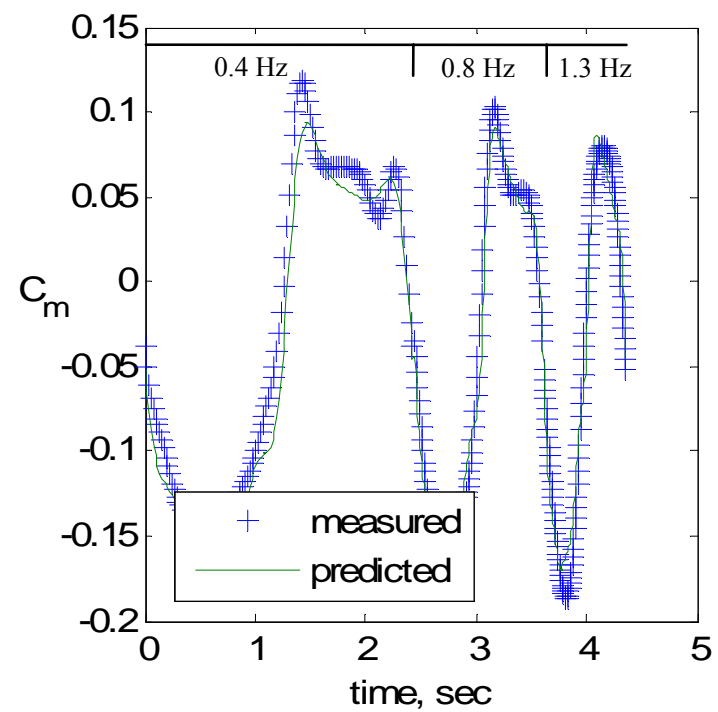

Figure 15. Measured and computed time histories of pitching moment for BW configuration, $\alpha_{0}=5^{\circ}$, and $\alpha_{A}=15^{\circ}$. 


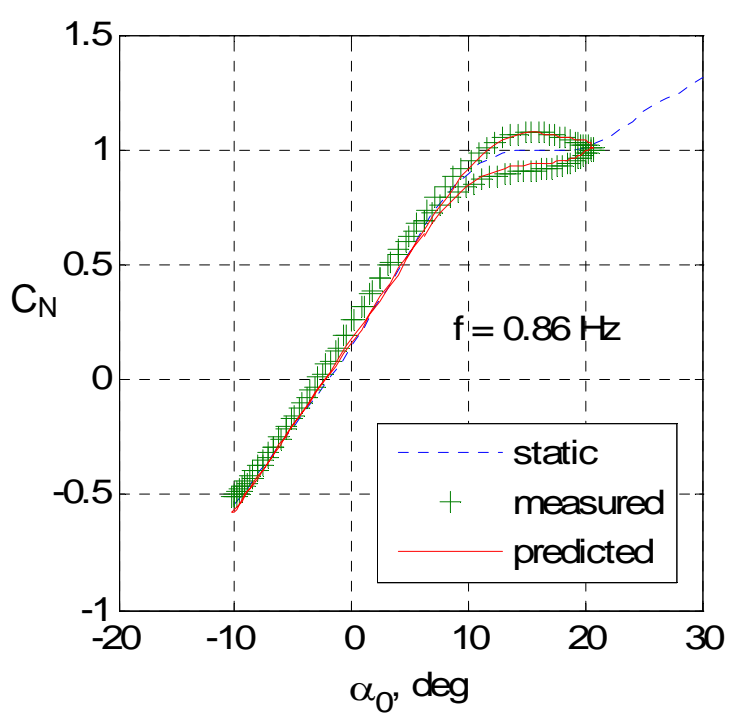

Figure 16. Variation of measured and computed normal force with $\alpha$ for BW configuration, $\alpha_{0}=5^{\circ}$, and $\alpha_{A}=15^{\circ}$.

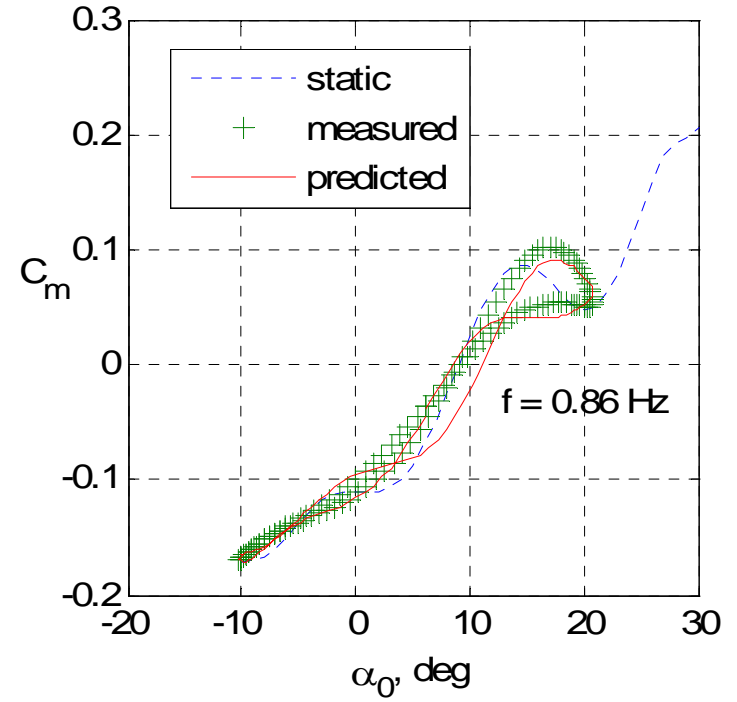

Figure 17. Variation of measured and computed pitching moment with $\alpha$ for BW configuration, $\alpha_{0}=5^{\circ}$, and $\alpha_{A}=15^{\circ}$.

\section{BWT Configuration}

As in the preceding section, model Eqs. (10-11) can be written with simplified notation giving

$$
\begin{aligned}
& \theta_{1} \dot{\eta}+\eta=u \\
& {\left[\begin{array}{l}
y_{1} \\
y_{2}
\end{array}\right]=\left[\begin{array}{l}
h_{1}(\eta) \\
h_{2}(\eta)
\end{array}\right]+\left[\begin{array}{l}
\theta_{2} \\
\theta_{3}
\end{array}\right] \frac{\dot{\alpha} \bar{c}}{2 V}}
\end{aligned}
$$

where

$$
\begin{aligned}
& \theta_{1}=\tau_{\varepsilon} \quad \theta_{2}=C_{N_{q}} \quad \theta_{3}=C_{m_{q}} \\
& \eta=\varepsilon \quad u=\varepsilon(\infty) \\
& h_{1}(\eta)=\bar{S}_{t} C_{N}\left(t ; \alpha_{t}\right) \\
& h_{2}(\eta)=-\bar{\ell}_{t} \bar{S}_{t} C_{N}\left(t ; \alpha_{t}\right)
\end{aligned}
$$

and outputs are defined as

$$
\begin{aligned}
& y_{1}=C_{N}(t ; \alpha)-C_{N}^{B}(\infty ; \alpha)-C_{N}^{W}(t ; \alpha) \\
& y_{2}=C_{m}(t ; \alpha)-C_{m}^{B}(\infty ; \alpha)-C_{m_{0}}^{W}-C_{N}^{W}(t ; \alpha) x^{W}(t ; \alpha)
\end{aligned}
$$

Nonlinear functions $h_{1}(\eta)$ and $h_{1}(\eta)$ are computed by integrating differential equations, Eqs.(28-29), using values of $\tau_{N}$ and $\tau_{x}$ estimated from BW forced oscillation data. As in the previous cases for this study, it was assumed that the unknown parameters are independent of $\alpha$.

Unknown parameters, $\theta=\left[\tau_{\varepsilon} C_{N_{q}} C_{m_{q}}\right]$, were estimated by stacking three sets of oscillatory data for each case defined by $\left[\alpha_{0}, \alpha_{\mathrm{A}}\right]$. Each set in the stack represented only one cycle of data at a single frequency. From the stacked data, $\tau_{\varepsilon}$ was estimated with an average value of 27 and a relatively high standard error of 5.6. Estimates 
for $C_{N_{q}}$ varied from -5 to 4 , and for $C_{m_{q}}$ values ranged from 14 to 24 . Negative values for $C_{N_{q}}$ and positive values for $C_{m_{q}}$ are unacceptable as follows from the previous discussions and physics of the problem.

Figures 18 and 19 compare measured and computed coefficients $C_{N}(t)$ and $C_{m}(t)$. The measured data were taken from three cycles at $\alpha_{0}=20^{\circ}$ and $\alpha_{A}=10^{\circ}$. The computed coefficients were based on parameter estimates $\tau_{\varepsilon}=26, C_{N q}=-5$, and $C_{m q}=14$. The agreement between measured and computed coefficients is very good.

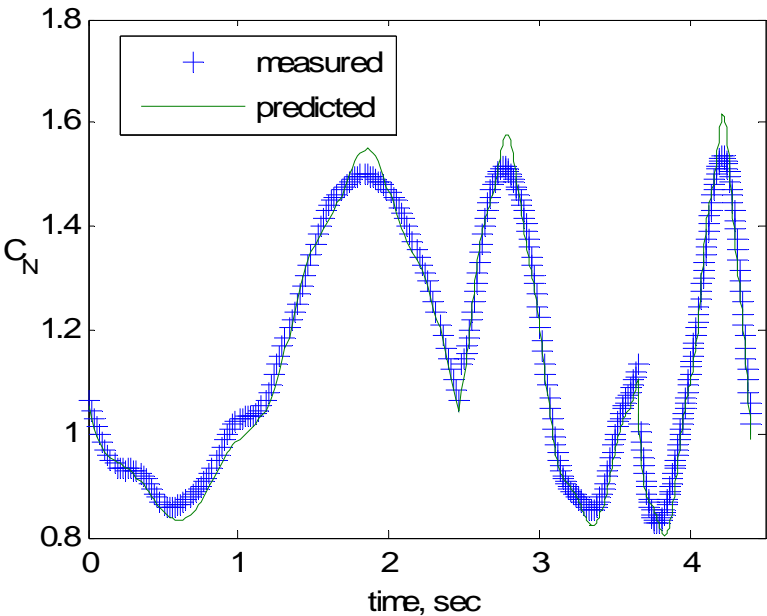

Figure 18. Measured and computed time histories of normal force for BWT configuration, $\alpha_{0}=20^{\circ}$, and $\alpha_{A}=10^{\circ}$.

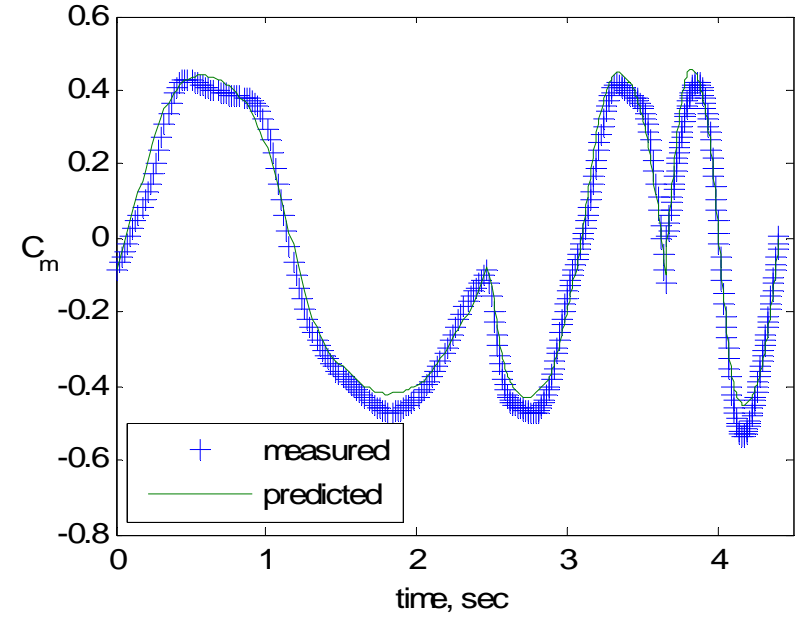

Figure 19. Measured and computed time histories of pitching moment for BWT configuration, $\alpha_{0}=20^{\circ}$, and $\alpha_{A}=10^{\circ}$.
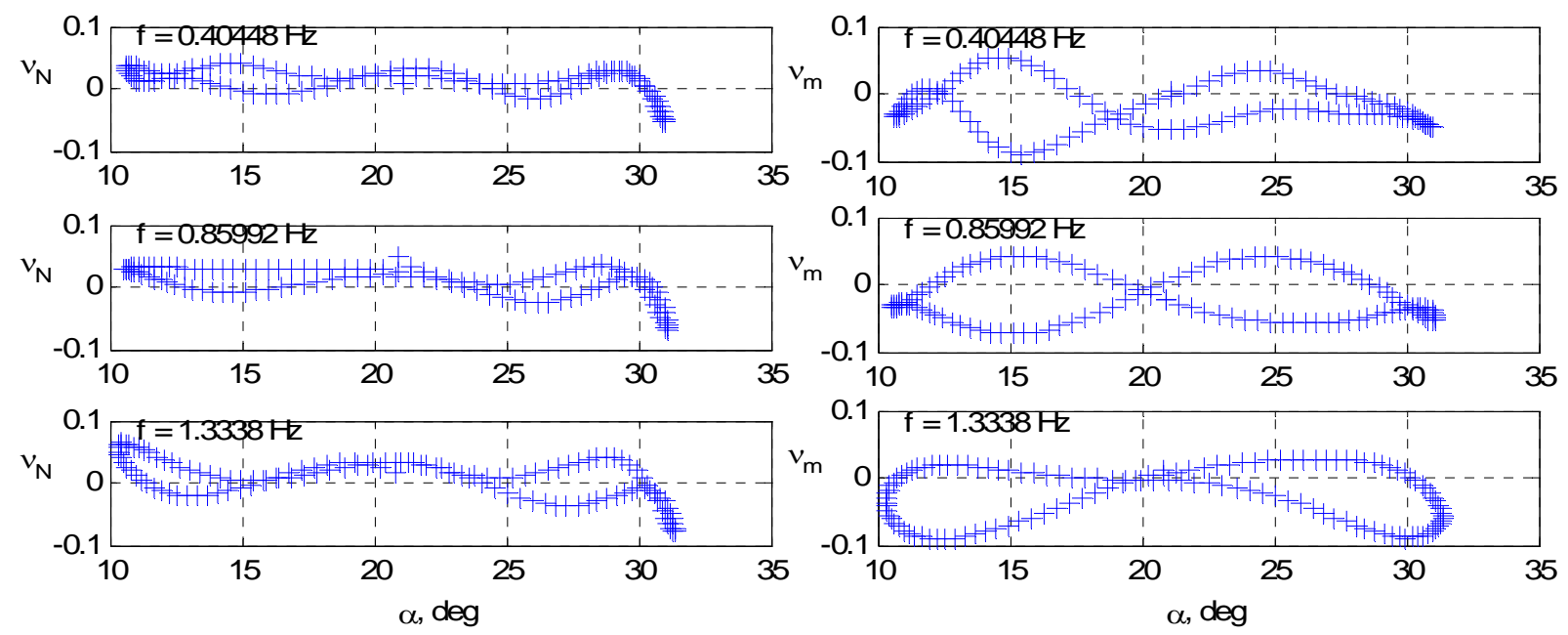

Figure 20. Residuals from measured and computed normal-force coefficient for BWT configuration, $\alpha_{0}=20^{\circ}$, and $\alpha_{A}=10^{\circ}$.

Figure 21. Residuals from measured and computed pitching moment coefficient for BWT configuration, $\alpha_{0}=20^{\circ}$, and $\alpha_{A}=10^{\circ}$.

The residuals plotted in Figs. 20 and 21 against $\alpha$ indicate deterministic errors in the data unexplained by the model equations. Ideally residuals should be zero mean and normally distributed. Strong insensitivity between model damping parameters, and total force and moment coefficient was also found in this case.

After parameter estimation for BWT configuration, an attempt was made to assess the prediction capabilities of the final model. The parameters in this model were selected as shown in the following table. 


\begin{tabular}{|c|c|c|}
\hline \multicolumn{2}{|c|}{ Parameter Specifications for Simulation } & Configuration \\
\hline$C_{N q}=5.1$ & $C_{m q}=-15.2$ & $\mathrm{BT}$ \\
\hline$\tau_{N}=13$ & $\tau_{x}=26$ & $\mathrm{BW}$ \\
\hline$\tau_{\varepsilon}=27$ & ----- & BWT \\
\hline
\end{tabular}

The coefficients $C_{N}(t)$ and $C_{m}(t)$ were computed for three frequencies, at $\alpha_{0}=20^{\circ}$ and $\alpha_{A}=10^{\circ}$, and compared with measured data. As in the previous case where $C_{N}(t)$ and $C_{m}(t)$ were estimated under the same conditions, the agreement between prediction and measurement was very good and the residuals were similar to those in Figs. 20 and 21 .

It is important to note that accurate assessment of model prediction must be based on data sets not used in parameter estimation. Validation data sets were not available in this case.

\section{Simulation Results}

Simulation results were used first, to ensure that all estimation algorithms were working properly and second, to assess a few experiment design issues that likely produced the strong insensitivity between parameter estimates and measurement data in this study. For brevity, only results from using the BW model given in Eqs. (25-29) and a limited number of test conditions are provided as examples. Test conditions demonstrate the effect of measurement noise, number of oscillation cycles, and model structure error in the form of measurement bias. A number of other experiment design factors are important for system identification of nonlinear unsteady systems but these are not considered in this paper. Discussion of frequency selection and other test design issues can be found in Refs. 4 and 14.

Model parameters were chosen to reflect a realistic set of values that are supported by the model identified in this paper. All simulation results are shown for a forced-oscillation experiment at $\alpha_{0}=15$ degrees and $\alpha_{\mathrm{A}}=15$ degrees. Two polynomial cases are demonstrated for the non-dimensional time constant, $\tau_{N}$, shown in Table III. In all simulation examples the starting values for parameter estimation are chosen to be $10 \%$ in error. This is a small starting error, but it is sufficient to demonstrate the estimation algorithms and specific test conditions of interest.

Table III. Simulation model parameters applied to BW configuration at $\alpha_{0}=15 \mathrm{deg}$ and $\alpha_{\mathrm{A}}=15 \mathrm{deg}$.

\begin{tabular}{|c|c|c|c|c|c|}
\hline Model Parameters & $\begin{array}{c}\text { Oscillation } \\
\text { Frequency }\end{array}$ & $\tau_{N}$ & $C_{N q}^{B W}$ & $\tau_{x}$ & $C_{m q}^{B W}$ \\
\hline Parameter Structure & $f(\mathrm{~Hz})$ & $c_{0}+c_{1} \alpha$ & $c_{0}$ & $c_{0}$ & $c_{0}$ \\
\hline \hline Case 1 (constant) & 0.8 & $15+0 \alpha$ & 0.5 & 20 & -2 \\
\hline Case 2 (linear poly) & 1.6 & $5+19.0986 \alpha$ & 0.5 & 20 & -2 \\
\hline
\end{tabular}

Case 1, with all parameters equal to constants, is an example demonstrating the efficacy of the estimation algorithms and the relative magnitude of the steady flow damping term, $C_{N q}^{B W}$. Figure 22 shows the results of using simulated measured data to estimate the normal force coefficient and predict its response against angle of attack. Model parameters were estimated exactly using only 1 oscillation cycle of data at $0.8 \mathrm{~Hz}$, providing some confidence in the estimation algorithms.

Because the steady flow damping coefficient, $C_{N q}^{B W}$, has a small value the damping term contributes relatively little to the response and consequently requires careful testing for successful model identification. For comparison, Fig. 23 shows the same model prediction as in Fig. 22 except only the steady flow damping term is present. The difference of the predicted response from the static curve is not easily visible demonstrating the very small contribution of the steady flow damping term. If $C_{N q}^{B W}$ is the sole term used to explain the response during model identification a very large and unrealistic value results indicating that this particular response can only be explained by a model with unsteady dynamics. 
The predicted response shown in Fig. 23 uses measured data for initial conditions to solve BW Eqns. (25-29), consequently the predicted response exhibits a small transient at $\alpha=15$ degrees. This response reflects the transition from the measured initial condition to the predicted steady harmonic motion.

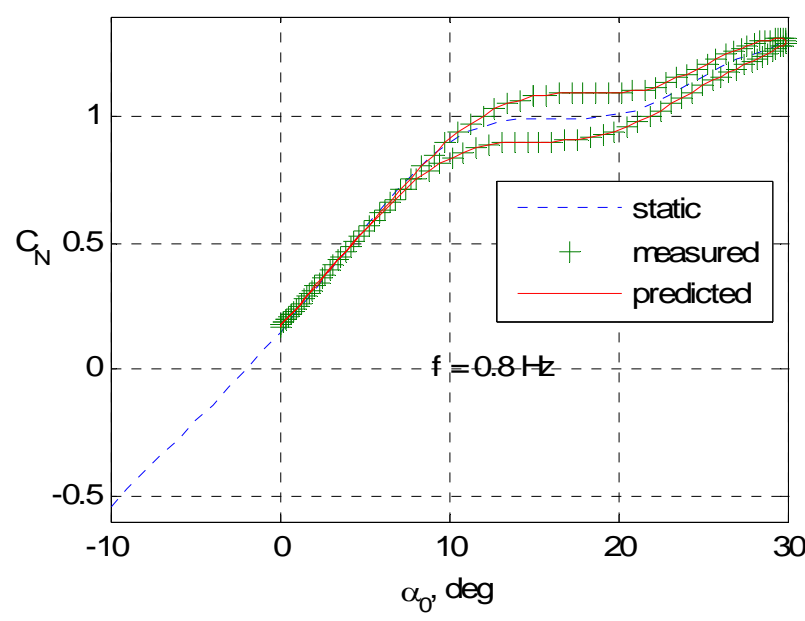

Figure 22. Variation of measured and computed normal force for BW configuration, at $\alpha_{0}=15^{\circ}$, and $\alpha_{A}=15^{\circ}$.

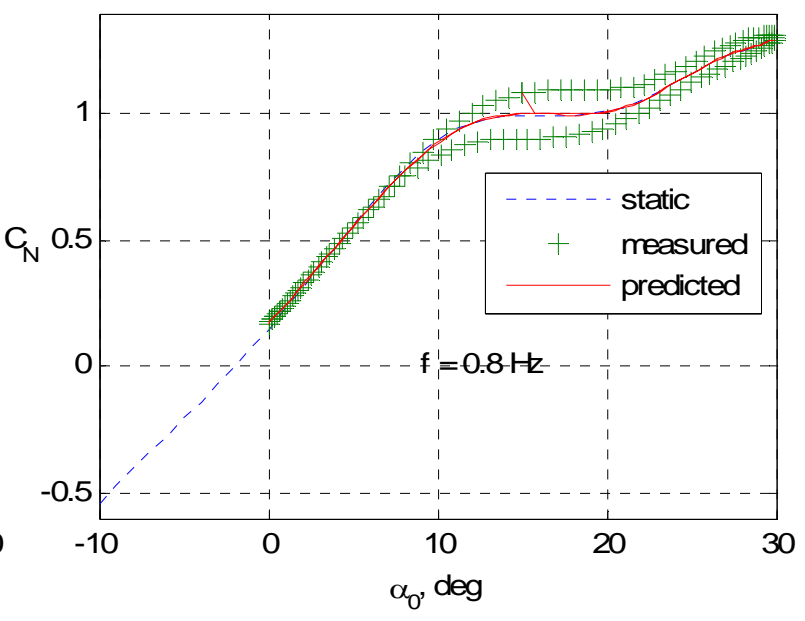

Figure 23. Variation of measured and computed normal force without unsteady model component for BW configuration, at $\alpha_{0}=15^{\circ}$, and $\alpha_{A}=15^{\circ}$.

To increase the realism of the estimation problem, possibly reflecting a change from steady to unsteady regions between low and high angle of attack, the unsteady time constant is allowed to vary with angle of attack. This is labeled as Case 2 in Table III. In this case the time constant is defined linearly from 5 to 15 over a range of angle of attack from $0^{\circ}$ to $30^{\circ}$. Initially only 1 cycle of oscillation data is used to simulate the real data case investigated in this paper. Two runs are made, one without noise and one with a very modest level of noise so that the signal to noise ratio is approximately 60. Measured and computed normal force coefficients are shown in Figs. 24 and 25 for this example. In both cases the estimated model predicts the response well. In the case with noise the parameters are estimated with extremely poor accuracy as shown in Table IV.

\begin{tabular}{|c|c|c|c|c|c|}
\hline \multicolumn{6}{|c|}{$\begin{array}{l}\text { Table IV. Estimated damping parameter and non-dimensional time constants } \\
\text { from simulated large-amplitude, 1-cycle oscillatory data, BW configuration at } f=1.6 \mathrm{~Hz}, \alpha_{0}=15, \alpha_{\mathrm{A}}=15 \mathrm{deg} \text {. } \\
\text { Parameter true values: } C_{N q}^{B W}=0.5, c_{0}=5, c_{1}=19.0986 \text {, where } \tau_{N}=c_{0}+c_{1} \alpha\end{array}$} \\
\hline Signal/noise & Fit error & \# cycles & $\hat{\theta}=\hat{C}_{N q}^{B W}$ & $\hat{\theta}=c_{0}$ & $\hat{\theta}=c_{1}$ \\
\hline & & & $\hat{\theta} \quad(\hat{\sigma})$ & $(\hat{\sigma})$ & $(\hat{\sigma})$ \\
\hline$\infty$ & $3.9 \mathrm{e}-06$ & 1 & $0.4997(0.0004)$ & $4.9995(0.0004)$ & $19.1001(0.0010)$ \\
\hline 60 & 0.017466 & 1 & $1.8424(1.8094)$ & $4.9343(1.6240)$ & $15.1883(4.6112)$ \\
\hline
\end{tabular}

The modest amount of noise masked the limited information content of the data and caused large parameter insensitivity. This is especially a problem for the very small steady flow damping term, $C_{N q}^{B W}$, in this case. To improve the model estimation in the presence of noise, the number of cycles of oscillation analyzed is increased and varied from 6 to 14 cycles. The parameter estimation results, shown in Table V, demonstrate, as expected, that increasing the number of oscillation cycles improves both parameter estimates and their standard errors. However, unexpectedly, the number of cycles required to improve parameter accuracy is relatively large compared to conventional practices. 


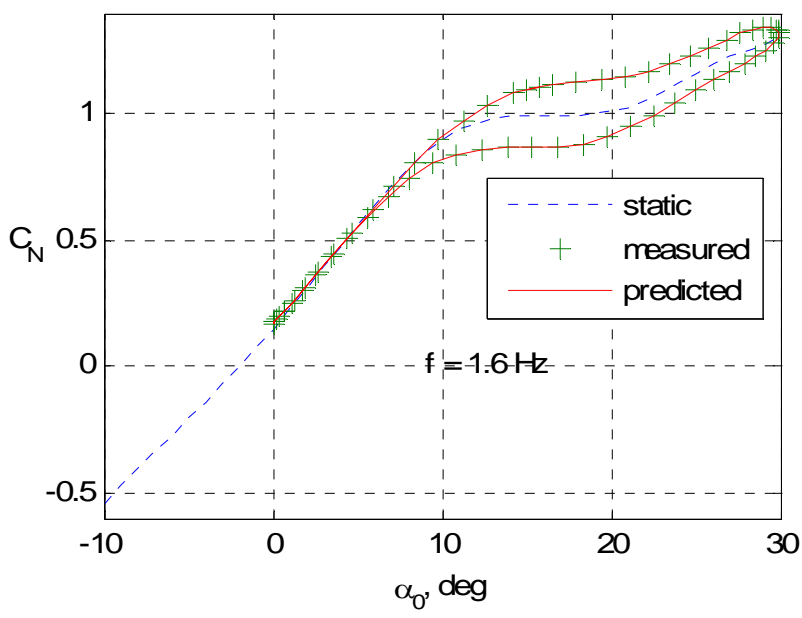

Figure 24. Variation of measured and computed normal force for BW configuration, at $\alpha_{0}=15^{\circ}$, and $\alpha_{A}=15^{\circ}$. Normal force time constant varies linearly with angle of attack. No noise is present.

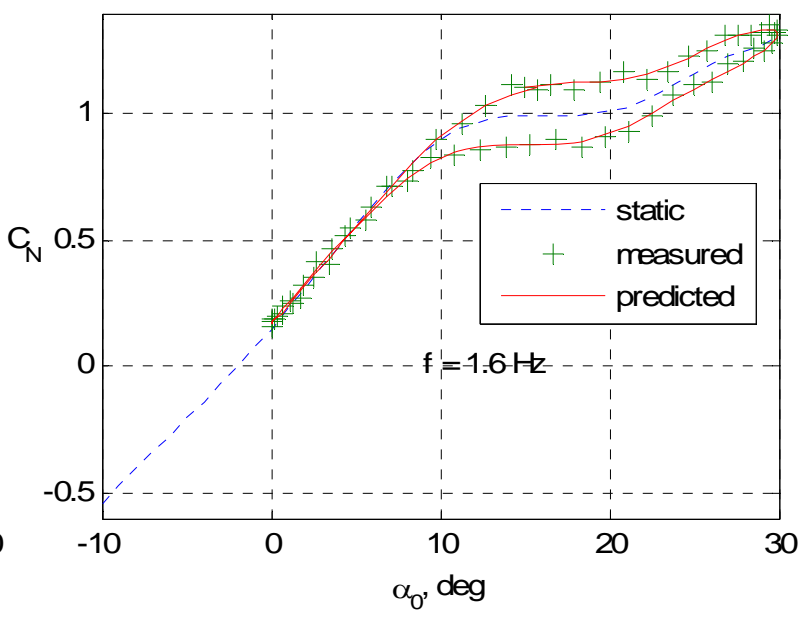

Figure 25. Variation of measured and computed normal force for BW configuration, at $\alpha_{0}=15^{\circ}$, and $\alpha_{A}=15^{\circ}$. Normal force time constant varies linearly with angle of attack. Signal/noise ratio $=60$.

\begin{tabular}{|c|c|c|c|c|c|}
\hline \multicolumn{6}{|c|}{$\begin{array}{l}\text { Table V. Estimated damping parameter and non-dimensional time constants } \\
\text { from simulated large-amplitude oscillatory data, BW configuration at } f=1.6 \mathrm{~Hz}, \alpha_{0}=15, \alpha_{A}=15 \mathrm{deg} \text {. } \\
\text { Parameter true values: } C_{N q}^{B W}=0.5, c_{0}=5, c_{1}=19.0986 \text {, where } \tau_{N}=c_{0}+c_{1} \alpha\end{array}$} \\
\hline Signal/noise & Fit error & \# cycles & $\hat{\theta}=\hat{C}_{N q}^{B W}$ & $\hat{\theta}=c_{0}$ & $\hat{\theta}=c_{1}$ \\
\hline & & & $\hat{\theta} \quad(\hat{\sigma})$ & $(\hat{\sigma})$ & $(\hat{\sigma})$ \\
\hline$\infty$ & $1.0 \mathrm{e}-06$ & 6 & $0.50(0.0001)$ & $4.999(0.0001)$ & $19.099(0.0002)$ \\
\hline 60 & 0.0158 & 6 & $0.3(0.68)$ & $4.5(0.62)$ & $20.7(1.75)$ \\
\hline 60 & 0.0158 & 9 & $0.6(0.55)$ & $4.6(0.50)$ & $20.0(1.43)$ \\
\hline 60 & 0.0155 & 14 & $0.5(0.43)$ & $4.8(0.40)$ & $19.7(1.12)$ \\
\hline
\end{tabular}

It is common practice in the aerodynamics field to apply one-factor at a time (OFAT) procedures when performing wind tunnel experiments. For example, typically, angle of attack is adjusted systematically over some range of interest. This lack of randomization can introduce systematic or bias errors into an experiment. To assess this issue simulated data was generated for 2 values of measurement bias error on the normal force coefficient. The bias errors were computed as a percentage of the maximum value of the normal force coefficient time history and the percentages were selected as $0.1 \%$ and $1 \%$. Table VI shows the estimation results when bias error is included for the same simulation considered in Table V. For each case with bias, 14 cycles of oscillation are used in the analysis. Estimation results using a model with bias error but without measurement noise are shown in the first three rows of Table VI. The results are revealing and indicate that even a very modest bias error can degrade estimation accuracy while a bias of $1 \%$ of maximum signal value confounds the estimation process, producing a non-physical mean value for the steady flow damping term. Estimates in the last 3 rows show that the modest bias error in combination with measurement noise significantly degrades the estimation accuracy and the $1 \%$ bias completely defeats the estimation process by preventing any convergence of the estimation algorithm. These results are similar to problems encountered with the real wind tunnel data in this study. 


\begin{tabular}{|c|c|c|c|c|c|}
\hline \multicolumn{6}{|c|}{$\begin{array}{l}\text { Table VI. Estimated damping parameter and non-dimensional time constants } \\
\text { from simulated large-amplitude oscillatory data, BW configuration at } f=1.6 \mathrm{~Hz}, \alpha_{0}=15, \alpha_{A}=15 \mathrm{deg} \text {. } \\
\text { Parameter true values: } C_{N q}^{B W}=0.5, c_{0}=5, c_{1}=19.0986 \text {, where } \tau_{N}=c_{0}+c_{1} \alpha\end{array}$} \\
\hline Signal/noise & Bias error & Fit error & $\hat{\theta}=\hat{C}_{N q}^{B W}$ & $\hat{\theta}=c_{0}$ & $\hat{\theta}=c_{1}$ \\
\hline & $(\% \max )$ & & $\hat{\theta} \quad(\hat{\sigma})$ & $\hat{\theta} \quad(\hat{\sigma})$ & $\hat{\theta} \quad(\hat{\sigma})$ \\
\hline$\infty$ & 0 & $1.0 \mathrm{e}-06$ & $0.50(0.0001)$ & $4.999(0.0001)$ & $19.099(0.0002)$ \\
\hline$\infty$ & 0.1 & 0.0013 & $0.46(0.036)$ & $4.8(0.03)$ & $19.8(0.094)$ \\
\hline$\infty$ & 1.0 & 0.0129 & $-0.1(0.36)$ & $2.9(0.33)$ & $26.5(0.94)$ \\
\hline 60 & 0 & 0.0155 & $0.5(0.43)$ & $4.8(0.40)$ & $19.7(1.12)$ \\
\hline 60 & 0.1 & 0.0154 & $0.4(0.43)$ & $4.7(0.40)$ & $20.6(1.11)$ \\
\hline 60 & 1.0 & $\sim$ & $\sim$ & $\sim$ & $\sim$ \\
\hline
\end{tabular}

\section{Concluding Remarks}

This paper first examines aircraft model identification from wind tunnel data of an airliner and its components: body, body-tail, body-wing, and the complete model. A mathematical model of an aircraft with structure sufficiently general to model nonlinear unsteady behaviors is considered. The measurement data included results from conventional static and one degree-of-freedom in pitch oscillatory tests over a wide range of angle of attack but limited frequencies and amplitudes.

All models with estimated parameters fit the measured data quite well. Damping parameters estimated from BT large-amplitude oscillation data were consistent with small-amplitude out-of-phase measurements and after including an $\alpha$-correlated term to correct for an inconsistency between normal force and pitch moment measurements, a very large percentage of the variation in the data was explained by the model. Damping parameters for the BW and BWT configurations were poor, however, the values were explained by a lack of parameter sensitivity or information content of the data.

Conventional forced-oscillation experiments generally focus on obtaining averaged single-cycle data at a limited number of frequencies. This approach facilitates a straightforward (sometimes graphical) approach for estimating out-of-phase damping in steady-flow linear regions. Identification of more complex models that capture nonlinear unsteady behaviors is not well suited for use with conventionally designed experiments as demonstrated by the very limited success of the BW and BWT model identification in this study. Simulated data were used to confirm the lack of parameter sensitivity in a conventional experiment and to demonstrate the potential severity of estimation error due to measurement noise and bias error. Although, theoretically, certain harmonic information is fully contained in a single cycle of data, both the actual and simulated results of this study support the need for a much greater number of cycles of data to overcome the effect of measurement error. This is especially true for situations where it is necessary to estimate model terms with relatively small magnitudes compared to the total aerodynamic force or moment.

\section{References}

${ }^{1}$ Goman, M. G., Stolyarov, G. I., Tyrtyshnikov, S. L., Usoltsev, S. P., and Khrabrov, A. N., "Mathematical Description of Aircraft Longitudinal Aerodynamic Characteristics at High Angles of Attack Accounting for Dynamic Effects of Separated Flow," TsAGI Preprint No. 9, 1990 (in Russian).

${ }^{2}$ Goman, M. G. and Khrabrov, A. N., "State-Space Representation of Aerodynamic Characteristics of an Aircraft at High Angles of Attack," Journal of Aircraft, Vol. 31, No. 5, 1994, pp. 1109-1115.

${ }^{3}$ Klein, Vladislav and Murphy, Patrick C., "Estimation of Aircraft Nonlinear Unsteady Parameters From Wind Tunnel Data," NASA TM-1998-208969, December 1998.

${ }^{4}$ Murphy, Patrick C. and Klein, Vladislav, "Validation of Methodology for Estimating Aircraft Unsteady Aerodynamic Parameters From Dynamic Wind Tunnel Tests," AIAA Atmospheric Flight Mechanics Conference, AIAA 2003-5397, AIAA Washington, DC, 2003.

${ }^{5}$ Klein, Vladislav, Murphy, P. C., and Szyba, Nathan M., "Analysis of Wind Tunnel Lateral Oscillatory Data of the F-16XL Aircraft," NASA TM-2004-213246, August 2004. 
${ }^{6}$ Smith, Mark S., “Analysis of Wind Tunnel Oscillatory Data of the X-31A Aircraft,” M.S. Thesis, The George Washington University, Washington DC, 1998.

${ }^{7}$ Jones, Robert T., "The Unsteady Lift of a Wing of Finite Aspect Ratio," NACA Report 681, 1939.

${ }^{8}$ Jones, Robert T. and Fehlner, Leo F., "Transient Effects of the Wing Wake on the Horizontal Tail," NACA TN-771, 1940.

${ }^{9}$ Tobak, Murray, "On the Use of Indicial Function Concept in the Analysis of Unsteady Motions of Wings and Wing-Tail Combinations, NACA Rep. 1188, 1954.

${ }^{10}$ Klein, Vladislav, "Modeling of Longitudinal Unsteady Aerodynamics at High Incidence with Account of Wing-Tail Interaction," NASA CR-1999-209547, September, 1999.

${ }^{11}$ Khrabrov, A., Vinogradov, Yu, and Abromov, N., "Mathematical Modeling of Aircraft Unsteady Aerodynamics at High Incidence with Account of Wing-Tail Interaction," AIAA Atmospheric Flight Mechanics Conference, AIAA 2004-5278, AIAA Washington, DC, 2004

${ }^{12}$ Murphy, Patrick C. and Klein, Vladislav, "Estimation of Longitudinal Unsteady Aerodynamics of a Wing-Tail Combination from Wind Tunnel Data," AIAA Atmospheric Flight Mechanics Conference, AIAA 2006-6154, AIAA Washington, DC, 2006.

${ }^{13}$ Etkin, Bernard, "Dynamics of Atmospheric Flight," John Wiley \& Sons, Inc., 1972.

${ }^{14}$ Murphy, Patrick C., Klein, Vladislav, and Szyba, Nathan M., "Progressive Aerodynamic Model Identification from Dynamic Water Tunnel Test of the F-16XL Aircraft," AIAA Atmospheric Flight Mechanics Conference, AIAA 2004-5277, AIAA Washington, DC, 2004. 